\title{
A Fast and Sensitive Method for the Simultaneous Determination and Quantification of Six Anionic Surfactants in Surface Water using HILIC-ESI- MS Technique
}

\author{
Upendra N. Dash and Saroj Kumar Paul* \\ Department of Chemistry, Institute of Technical Education and Research (ITER), Siksha 'O' Anusandhan University, \\ Bhubaneswar, Odisha, India
}

Received August 9, 2012; Revised September 3, 2012; Accepted September 3, 2012

First published on the web September 20, 2012; DOI: 10.5478/MSL.2012.3.3.78

\begin{abstract}
The hydrophobic hydrocarbon chain and the polar sulfate group confer surfactant properties and enable them to be used as anionic surfactants. Anionic surfactants (AS) are known for their adverse impact on environment, particularly on aquatic ecosystem. In the present study a fast, sensitive and selective method for the determination and subsequent quantification of six anionic surfactants was developed using hydrophilic interactive liquid chromatography (HILIC) coupled to a electrospray ionization (ESI) mass spectrometer (MS), in the concentration range $15-20 \mu \mathrm{g} / \mathrm{L}$. The capability of the method was established using regression analysis and ANOVA. The method performance was evaluated by analyzing real time surface water spiked with 1-dodecyl hydrogen sulfate at $15 \mu \mathrm{g} / \mathrm{L}$. Combined efficiency of solid phase extraction and MS detection established recovery of $89 \%$ in presence of natural matrix. These results point out that HILIC coupled to multistage MS procedures can be a powerful technique for environmental applications concerning the screening of polar contaminants.
\end{abstract}

Keywords : Water pollution, Anionic surfactants, HILIC, LC MS/MS

\section{Introduction}

One of the key environmental problems of $21^{\text {st }}$ century is the increased worldwide contamination of fresh water ecosystems with numerous natural and industrial chemicals. Synthetic surface-active substances (surfactants) are produced worldwide in large volumes. The total world consumption of surfactants in 2003 was estimated at approximately 9.2 million tons, 4.5 million tons of which was from the consumption of anionic surfactants. ${ }^{1}$

Anionic surfactants consist of a negatively charged polar group and a hydrophobic alkyl group. They are largely used in cleaning applications owing to their ability to lower down the surface tension of water and currently used in shampoos, hard surfaces cleaners, bath preparations, cosmetics, medicines, toothpastes and laundry applications. Most of the surfactants are toxic to the aquatic ecosystem due to their surface activity. G. Konnecker et al. reviewed a large number aquatic toxicity studies performed on different classes of anionic surfactants including alkyl sulfates. ${ }^{2,3}$ Studies related to the interfacial properties have been reported in literature which evidently justify the hypothesis that toxicity of surfactants is determined by adsorption on biological membrane and cell membrane penetration. ${ }^{4}$

*Reprint requests to Prof. Saroj Kumar Paul

E-mail: paul.saroj@gmail.com
Due to their widespread use and toxicity, several analytical techniques have been reported in the literature for the effective screening of AS in water bodies. ${ }^{5,6}$

Generally, analytical methods for the quantitative determination of surfactants must be fast, accurate and costeffective for routine application. The most widely used technique for the industrial control of anionic surfactants of detergents in routine procedures is based on the two-phase titration method, ${ }^{7,8}$ which suffers from many drawbacks. They are time-consuming, tedious procedures that are not suitable for routine measurements, and they require the use of chloroform, which is restricted for environmental and toxicological reasons. These non specific methods are also subject to interferences from non surfactant organic sulfates leading to error in estimation.

However owing to their non-volatile characteristic liquid chromatography based techniques are predominately gaining importance for the monitoring of $\mathrm{AS}$, nevertheless adequate amount of spectrophotometric and other non specific methods are also there in literature. ${ }^{9}$ However absence of chromophoric groups requires LC methods to make use of derivatization which though renders sensitivity but not completely devoid of interference from waste water matrices and also demands longer time for carrying out the dervatization steps.

The fact that the classical reverse phase high performance liquid chromatography (HPLC) stationary phase requires high amount of aqueous and ionic pair based eluent to 
Table 1. List of anionic surfactants with their structure

\begin{tabular}{|c|c|c|c|c|}
\hline Sr. No. & IUPAC Name & Structure & Exaxct mass & Assigned code \\
\hline 1 & pentane-1 -sulfate & & 152.05 & AS-1 \\
\hline 2 & 1-hexane sulfate & & 165.06 & AS-2 \\
\hline 3 & 1-heptane sulfate & & 179.07 & AS-3 \\
\hline 4 & 1-octane sulfate & & 193.09 & AS-4 \\
\hline 5 & 1-decane sulfate & & 221.12 & AS-5 \\
\hline 6 & 1-dodecyl hydrogen sulfate & & 265.15 & AS-6 \\
\hline
\end{tabular}

resolute different AS components in a matrix, led to appreciable suppression of ionization and false negative outcomes in ESI-MS studies. The aim of this study was to determine the concentration of six homologous alkyl and alkane sulfates as shown in Table.1, in water using a HILIC column that make use of zwitterionic stationary phase to cause separation of polar AS molecules. In addition the increased affordability to use high concentration of organic eluent without affecting the desired retention behaviour of AS components also enhanced the sensitivity of the ESIMS to evidently lower down the detection limit(DL).The applicability of this methodology was also established by analysing the real time surface water for the screening of AS. Use of HILIC-ESI-MS technique not only enhanced the sensitivity but also reduced the analysis time by eliminating the dervatization procedures.

To the best of our knowledge such elaborate study on the homologous AS using HILIC-ESI-MS hasn't been reported till date.

\section{Experimental}

\section{Reagents, Standard solutions and sample collection}

Individual standards of the sodium salts of pentane-1sulfonic acid, 1-hexane sulfonic acid, 1-heptane sulfonic acid, 1-octane sulfonic acid, 1-decane sulfonic acid and 1dodecyl hydrogen sulfate were obtained from spectrochem (Mumbai, India). De-ionized reagent water was prepared using a Milli-Q plus water purification system from Millipore (Bradford, Pennsylvania, USA). HPLC grade methanol and acetonitrile were purchased from Merck India Limited (Mumbai, India).Analytical reagent grade ammonium acetate and formic acid were purchased from Qualigens India Limited (Mumbai, India).sample of surface water was collected from water bodies close to the city of New
Delhi, India. An Oasis HLB cartridge (Waters Corporation, MA, USA) was used for enrichment of surface water.

\section{Chromatographic and mass spectrometry}

The MS and MS/MS studies were performed on 3200 Qtrap mass spectrometer (AB Sciex, CA, USA) using electrospray ionization source (negative polarity).The instrument was operated in multiple reaction monitoring mode with the following settings: collision energy of $40 \mathrm{~V}$, collision energy spread $10 \mathrm{~V}$ declustering potential $10 \mathrm{~V}$ and the capillary ion spray voltage was $4500 \mathrm{~V}$. Nitrogen was used as curtain gas and CAD gas at a pressure of 15 psi. Zero air at a pressure of 45 psi was used as nebuliser gas and heater gas. The molecular ion of the AS-1, AS-2, AS-3, AS-4, AS-5 and AS-6 were observed at m/z 151.2, 165.3, $179.3,193.3,221.3$ and 265.3 respectively as depicted in Figure 1 and produced key fragment ions at $\mathrm{m} / \mathrm{z} 80$ for compounds AS- 1 to AS-5 and m/z 95 for AS-6. Simultaneous monitoring of daughter ions and the respective molecular of the six AS were done for increased specificity and accuracy. The instrument response was optimized for individual AS

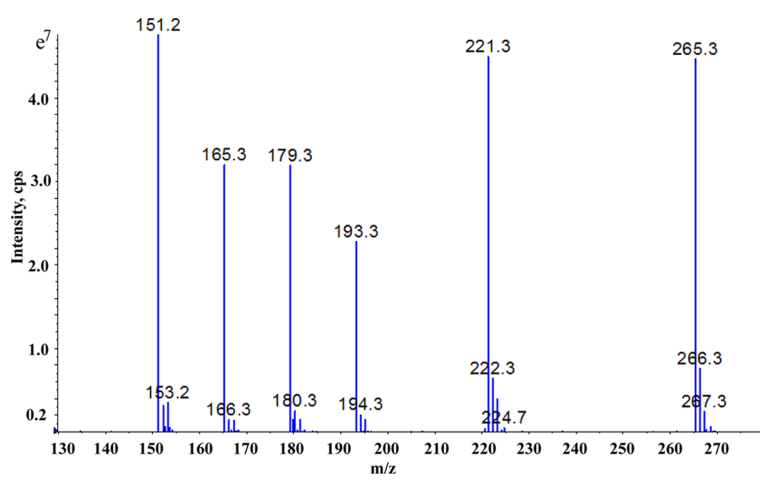

Figure 1. Representive mass spectrum of the mixture of six AS. 


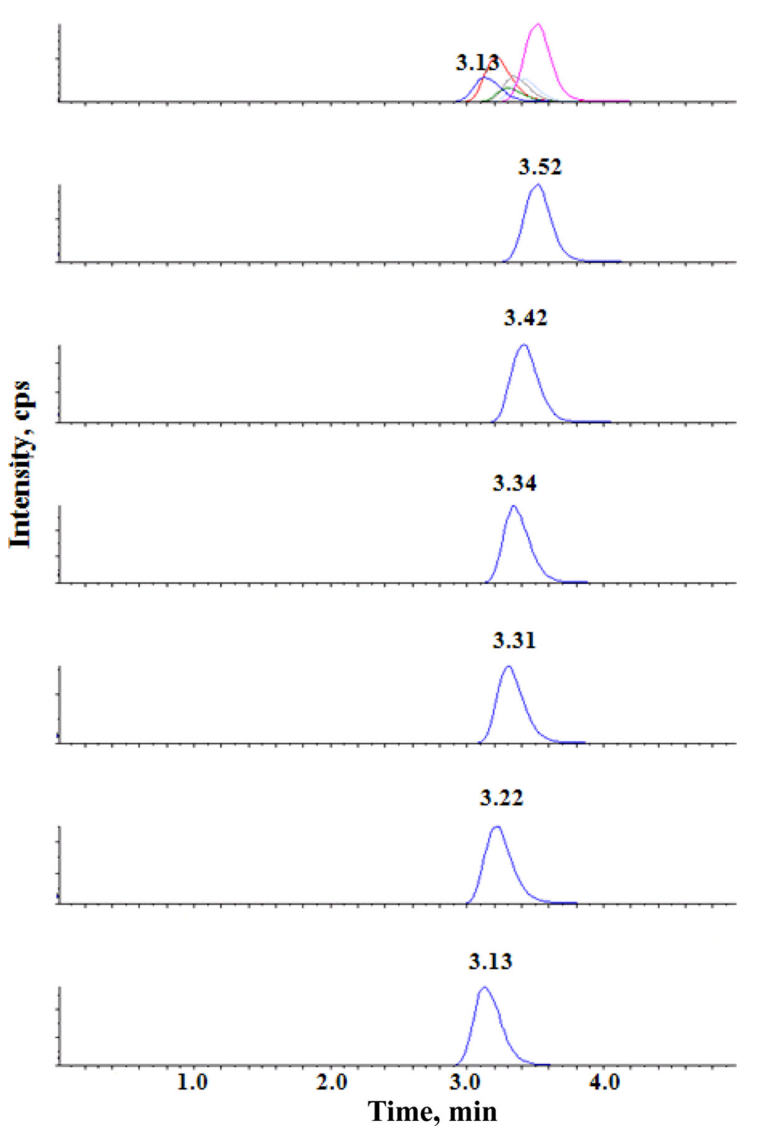

Figure 2. Representive extracted ion chrmatogram of the mixture of six AS.

by infusing a constant flow of respective standard solutions (1000 ng mL-1).

The HPLC consisted of LC-20AD binary gradient pump, SPD-10AVP UV detector, SIL-10HTC auto sampler and a column oven CTO-10ASVP (Shimadzu Corporation, Kyoto, Japan). A ZIC HILLIC column (Merck, Darmstadt, Germany, $100 \times 4.6 \mathrm{~mm}, 5 \mu \mathrm{m}, 200 \AA$ ) was used for chromatographic separation. Mobile phase A consisted of $10 \mathrm{mM}$ ammonium acetate, $\mathrm{pH}$ adjusted to 6 with formic acid and mobile phase $\mathrm{B}$ consisted of acetonitrile $(35: 65 \mathrm{v} /$ v) in isocratic mode. The injection volume was $20 \mu \mathrm{L}$, the flow rate was kept constant at $0.6 \mathrm{~mL} / \mathrm{min}$ and the temperature of the column was set to $30{ }^{\circ} \mathrm{C}$. The same eluent was also used for preparing the final solution of the standards of the six anionic surfactants listed in Table 1.

An extracted ion chromatogram showing the six anionic surfactants is given in Figure 2.

Water sample collected was divided into two different container of one litre each. One part was spiked with AS-6 in the range $15 \mu \mathrm{g} / \mathrm{kg}$ while the other part left untreated to be used as blank. Prior to spiking, the $\mathrm{pH}$ of the water sample was measured to be at 5.4. The measurement of $\mathrm{pH}$ was done to establish that water taken for analysis is not highly alkaline or acidic as extreame $\mathrm{pH}$ may affect the efficiency ion exchange catridge. Both the samples were filtered for solid particles and preconcentrated using an offline approach onto Oasis catridge. $5 \mathrm{~mL}$ of methanol and $5 \mathrm{~mL}$ of deionized water passed sequentially to condition the cartridge followed by $1 \mathrm{~L}$ of sample water. Finally the compounds trapped on the sorbent were eluted with $5 \mathrm{~mL}$ of methanol and $5 \mathrm{~mL}$ of deionized water containing $0.1 \%$ formic acid. Each of these extracts were reduced to $100 \mu \mathrm{L}$ under nitrogen and brought up to volume of $1 \mathrm{~mL}$ with the aforesaid HPLC eluent. The concentrated solution was filtered through $0.22 \mu \mathrm{m}$ GHP filter and analyzed by HILICESI-MS under aforementioned optimized conditions.

\section{Validation studies}

Each of the six AS were separately prepared in milli-Q water and then diluted to the desired concentration levels. The applicability of the method was established by studying the following parameters.

\section{Limit of detection (DL) and quantification (QL)}

DL and QL were determined on the basis of response. The lowest concentration which gives $\mathrm{s} / \mathrm{n}$ value of 3 and 10 were determined by injecting individual dilute standards of AS in the ESI-MS which then assigned to be the DL and $\mathrm{QL}$ respectively.

\section{Linearity}

The chromatographic linearity range was studied by performing triplicate analysis of AS solutions over 5 concentration range from 50 to $150 \%$ of the QL limit. The linearity test solutions were prepared by diluting the

Table 2. Summary of validation study

\begin{tabular}{|c|c|c|c|c|c|c|}
\hline \multirow{2}{*}{$\begin{array}{l}\text { Compound } \\
\text { name }\end{array}$} & \multirow{2}{*}{$\mathrm{DL}$ in $\mu \mathrm{g} / \mathrm{L}$} & \multirow{2}{*}{$\mathrm{QL}$ in $\mu \mathrm{g} / \mathrm{L}$} & \multirow{2}{*}{$\begin{array}{l}\text { Precision at QL } \\
\quad(\% \text { RSD })\end{array}$} & \multicolumn{3}{|c|}{ Linearity } \\
\hline & & & & $\mathrm{P}$ & Slope & Intercept \\
\hline AS-1 & 2 & 4 & 0.81 & 1.0000 & 357689 & 241 \\
\hline AS-2 & 4 & 6 & 1.23 & 0.9998 & 426950 & 545 \\
\hline AS-3 & 3 & 5 & 5.13 & 0.9996 & 246506 & 1120 \\
\hline AS-4 & 2 & 5 & 2.65 & 0.9995 & 376437 & 2073 \\
\hline AS-5 & 4 & 6 & 1.44 & 0.9975 & 205712 & 2404 \\
\hline AS-6 & 3 & 5 & 3.34 & 0.9979 & 227945 & 2401 \\
\hline
\end{tabular}


individual standard solutions into single mixtures with resultant concentration adjusted to their respective QL value.

\section{Accuracy}

The accuracy of this method was checked by percent recovery method at QL level. The recoveries were determined by spiking surface water with mixed standard solution of the six AS adjusted to their respective QL value which followed the procedure of solid phase extraction and enrichment as described in the section on chromatography and mass spectrometry.

\section{Precision}

The precision and repeatability of the method was evaluated by analyzing the AS solution six times at QL level to determine the percentage of relative standard deviation $(\% \mathrm{RSD})$ of the area under the peak at repeated analysis.

\section{Result and Disscussion}

The HILIC-ESI-MS method developed for the determination and quantification of six homologous AS found to show acceptable recoveries and reproducibility over a range of concentrations. The initial attempted trails with classical reverse phase C18 HPLC columns didn't produce the required resolution using MS friendly eluent combinations of $2-5 \mathrm{mM}$ ammonium acetate in a $40-90 \%$ gradient of acetonitrile, for specific and sensitive determination of the mixture of six homologous alkyl and alkane sulfates. Wide peaks and tailing were observed. However use of zwitterionic stationary phase based HILIC column offered adequate resolution and retention eliminating the need to use high concentration of non volatile buffer or ion pairing modifier. The mutual interaction of polar sulfate group with stationary phase was identical to all the compounds, where as varying the alkyl chain lengths enabled them to interact differently with organic eluent containing $80 \%$ acetonitrile. High concentration of organic phase in the elutent also helped to achieve lower DL and QL as they help in droplet formation in electrospary ionization and have an edge over highly aqueous mobile phase. ${ }^{10}$ The method was validated with respect to Linearity, accuracy and precision. Regression statistics and analysis of variance was employed to establish the linearity of the responses of AS compounds over the range of concentrations. The results of the method validation is given in Table 2 .

The precision was studied by analysing six samples at each concentration level. The $\%$ of relative standard deviation obtained was within the acceptable limit.

ANOVA studies carried out also indicated significant linearity with F-ANOVA at 1145.845627

The applicability of the method was also established by analysing surface water containing natural matrix and spiked with known concentration $(15 \mu \mathrm{g} / \mathrm{kg})$ of AS-6 to investigate possible matrix interference in solid phase extraction and MS analysis. However mean recovery of $89 \%$ established the efficiency of the method for analysis of surface water to monitor the anionic surfactants.

The apparently shorter analysis time will also enable researchers for quick decision making while investigating the environmental fate of anionic surfactants with respect to their biodegrability and interaction with other chemical entities.

\section{Conclusions}

A new fast, sensitive and selective method was developed for the simultaneous determination and quantification of six homologous anionic surfactants in surface water using hydrophilic interactive liquid chromatography coupled to an electrospray mass spectrometer with quadraouple mass analyzer. The method performance was evaluated with respect to linearity, accuracy and precision. Satisfactory recovery was also obtained while examining real sample spiked with known concentration of AS and hence established the applicability of the method to monitor the anionic surfactant content in surface water for studying their fate in water bodies as well as impact on biotic ecosystem.

\section{Acknowledgment}

The authors would like to thank Mr. Sabyasachi Rout of BARC, Mumbai for his support in carrying out the statistical analysis.

\section{References}

1. Brackmann, B.; Hager, C. D. Proceedings, 6th World Surfactant Congress CESIO, Berlin (Germany), 21-23 june, 2004.

2. Tibor, C.; Esther, F. ; Gyula, O. Environment International, 2002, 21, 337

3. Konnecker, J. G.; Regelman, S.; Belanger, K.; Gamon and Sedlak, R. Ecotoxicology and Environmental Safety, 2011, 74, 1445

4. Rosen, M. Environmental Science \& Technology, 2001, 35,954

5. Ventura, F.; Caixach, J.; Romero, J.; Espadaler, I.; Rivera, J. Journal of water science and technology, 1992, 25, 257

6. Richardson, S. D. Chem. Rev. 2001, 101, 211

7. URL:http://www.firp.ula.ve/archivos/material_web_4xx/ 04 CESIO Brackmann 112.

8. Epton, S. R. Trans. Faraday Soc. 1948, 44, 226.

9. Issei, K.; Kanae, H.; Tomoko, K.; Akiko, K.; Keiji, M.; Noriko, H.; Shigeru, T.; Katsumi, G. Analyst, 1995, 120 1803.

10. Kebarle. P.; Tang, L. Anal. Chem. 1993, 65, 972A. 